\title{
Aferesi nel rigetto acuto del rene trapiantato
}

\author{
Giovanni M. Frascà, Emilio Balestra, Domenica Taruscia, Valentina Nastasi, \\ Giovanni Gaffi, Mariastefania Pugliese
}

Nefrologia, Dialisi e Trapianto di rene, Ospedali Riuniti, Ancona

\begin{abstract}
APHERESIS IN THE TREATMENT OF ANTIBODY-MEDIATED ACUTE RENAL GRAFT REJECTION
Abstract. Basic indications for the use of apheresis in the treatment of antibody-mediated acute rejection include: (i) resistance to conventional anti-rejection therapy; (ii) poor prognosis for the graft; (iii) the need for a rapid removal of antibodies from the circulation, since their half-life is of about 3 weeks.

Despite the large number of cases already reported in the literature, the role of apheresis in the treatment of acute rejection is still a matter of discussion. This is due to the difficulties in drawing definitive conclusions on its effectiveness for the lack of large controlled trials as well as for the variability in treatment schedules and the associated drug therapy. However, in many transplant centers apheresis is currently used to treat antibody-mediated acute rejection.

The criteria for an appropriate use of apheresis in these settings are: (i) a prompt diagnosis of antibody-mediated rejection by means of graft biopsy; (ii) starting apheresis as soon as possible after diagnosis, and before irreversible damage of the graft occurs, without waiting for failure of drug therapy; (iii) the association with immunosuppressive agents effective on antibody production.
\end{abstract}

Key words: Apheresis, Antibody-mediated rejection, Plasmaexchange, Renal transplantation

Conflict of interest: None.

Financial support: None.

Ricevuto: 13 Gennaio 2013; Accettato: 20 Febbraio 2013

Il trapianto renale è ormai entrato nella pratica clinica corrente e rappresenta attualmente il trattamento migliore dell'insufficienza renale cronica, in termini di mortalità, morbidità e qualità di vita. L'incidenza del rigetto acuto del trapianto, che ha rappresentato un problema clinico rilevante prima dell'introduzione degli inibitori della calcineurina, si è progressivamente ridotta con il passare degli anni, grazie alla disponibilità di nuovi farmaci immunosoppressori e alla terapia di induzione con anticorpi anti timociti (ATG) o anticorpi contro il recettore dell'interleuchina-2 (anti-CD25), ormai di uso corrente.

Con gli schemi di terapia attualmente utilizzati, il rigetto acuto si verifica mediamente nel $10 \%$ circa dei pazienti sottoposti a trapianto. Malgrado ciò, ancora oggi le cause immunologiche rappresentano uno dei principali motivi di fallimento del trapianto (1).

\section{Meccanismi immunologici del rigetto acuto del trapianto renale}

È noto da tempo che il rigetto acuto del rene trapiantato può essere sostenuto da meccanismi immunologici diversi, a seconda che coinvolgano prevalentemente o esclusivamente meccanismi immunologici di tipo cellulo-mediato o anticorpo-mediato, questi ultimi sostenuti da anticorpi diretti contro antigeni di istocompatibilità del donatore 0 contro altri antigeni espressi dall'endotelio.

Ai diversi meccanismi immunologici alla base del rigetto corrispondono lesioni istologiche diverse (2) che, nel primo caso, consistono prevalentemente in un infiltrato infiammatorio tubulo-interstiziale, con un'evidente tubulite e una quota variabile di necrosi tubulare, mentre, nel rigetto mediato da anticorpi, prevalgono le lesioni vascolari, con endoarterite e infiltrazione della parete arteriolare, fino alla necrosi fibrinoide, alle quali si associa spesso la presenza di linfociti nel lume dei capillari glomerulari ("glomerulite"). Nei casi più severi, la reazione degli anticorpi con gli antigeni presenti sull'endotelio e la successiva attivazione del complemento attivano i meccanismi della coagulazione. Lo studio immunoistochimico dimostra generalmente la presenza della frazione $\mathrm{C} 4 \mathrm{~d}$ del complemento sui capillari peritubulari (2), anche se è possibile osservare lesioni di tipo vascolare anche in assenza di depositi di C4d (3). 


\section{Presupposti per l'impiego della terapia aferetica nel rigetto acuto del rene trapiantato e risultati}

I presupposti per l'impiego della terapia aferetica nel trattamento del rigetto mediato da anticorpi sono rappresentati: a) dalla prognosi nettamente peggiore rispetto a quella del rigetto cellulo-mediato (4), b) dalla scarsa o assente risposta alla terapia antirigetto tradizionale, con boli di steroidi e c) dalla possibilità di rimuovere rapidamente dal circolo anticorpi e mediatori dell'infiammazione (5). Studi effettuati all'inizio degli anni ' 80 avevano dimostrato che l'aferesi è in grado di rimuovere rapidamente dal circolo gli anticorpi responsabili del rigetto, migliorando la funzione del trapianto (5). Queste osservazioni erano state confermate successivamente da uno studio controllato, nel quale 23 pazienti trattati con aferesi erano stati confrontati con 21 pazienti trattati solo con terapia farmacologica (6), con una sopravvivenza del trapianto statisticamente significativa già a 3 mesi $(70 \%$ dei pazienti trattati con aferesi contro $20 \%$ del gruppo di controllo), e da un'analisi dei risultati a distanza (7).

Tuttavia, nonostante il passare degli anni, esiste ancora una certa discussione sull'efficacia dell'aferesi nel trattamento del rigetto acuto (8), per la difficoltà di trarre conclusioni dai numerosi studi riportati, legata a molteplici fattori che includono: a) la presenza di molti studi aneddotici per la difficoltà di effettuare studi controllati su ampie casistiche, b) la grande variabilità degli studi riportati in letteratura per quanto riguarda i criteri utilizzati nel porre la diagnosi di rigetto mediato da anticorpi e c) la difformità nelle modalità di trattamento, in termini di inizio del trattamento, frequenza, numero di sedute, liquido di reinfusione e, soprattutto, terapia associata.

Il trattamento del rigetto acuto anticorpo-mediato, infatti, non può prescindere dall'impiego di una terapia efficace nel bloccare la produzione anticorpale. In passato è stata utilizzata a questo scopo la ciclofosfamide (7), associata, tuttavia, a un'elevata incidenza di complicanze infettive e attualmente sostituita dall'infusione di gamma globuline ad alto dosaggio o dal rituximab (anti-CD20) (9). Più recentemente, è stato proposto l'uso del bortezomib, inibitore del proteasoma, che dovrebbe essere in grado di bloccare con maggiore efficacia la produzione anticorpale per la sua azione sulle plasmacellule che il rituximab non possiede (10).

Comunque, dal momento che l'emivita degli anticorpi è di circa 3 settimane, è evidente la necessità di neutralizzarli e di rimuoverli prima che i danni all'organo trapiantato diventino irreversibili, anche se in presenza di una terapia immunodepressiva efficace.

\section{Conclusioni}

L'aferesi è uno strumento indispensabile per il trattamento del rigetto acuto mediato da anticorpi. I criteri per il suo corretto utilizzo comprendono:

- una diagnosi tempestiva di rigetto anticorpo-mediato mediante una biopsia del trapianto;
- l'inizio tempestivo dell'aferesi senza attendere il fallimento della terapia farmacologica, una volta posta la diagnosi, per evitare lesioni estese e potenzialmente irreversibili del rene trapiantato;

- l'associazione con una terapia adeguata per bloccare la produzione anticorpale.

\section{Riassunto}

I presupposti per l'impiego dell'aferesi nel trattamento del rigetto acuto del rene trapiantato mediato da anticorpi comprendono: a) la scarsa o assente efficacia della terapia antirigetto tradizionale, b) la prognosi sfavorevole per il trapianto $\mathrm{e}$ ) la necessità di rimuovere rapidamente dal circolo gli anticorpi, in attesa che gli interventi mirati a bloccarne la sintesi abbiano effetto. Tuttavia, nonostante l'aferesi sia utilizzata da diversi anni e la letteratura riporti un'ampia casistica, è ancora oggetto di discussione se questa abbia un ruolo nel trattamento del rigetto acuto del rene trapiantato. La ragione risiede nella mancanza di studi controllati di ampie dimensioni per l'obiettiva difficoltà che questa patologia comporta, nei diversi criteri utilizzati per la diagnosi di rigetto e nella variabilità degli schemi di trattamento e della terapia farmacologica utilizzati nei vari studi. Malgrado ciò, molti centri trapianto utilizzano attualmente l'aferesi per il trattamento del rigetto acuto mediato da anticorpi.

I criteri per l'impiego dell'aferesi in questi casi comprendono: 1) la diagnosi tempestiva di rigetto mediato da anticorpi mediante una biopsia del rene trapiantato, 2) l'inizio tempestivo del trattamento con aferesi prima che si instaurino lesioni irreversibili del trapianto e 3) l'associazione con una terapia farmacologica in grado di bloccare la produzione anticorpale.

Parole chiave: Aferesi, Rigetto anticorpo-mediato, Plasmaferesi, Trapianto renale

Dichiarazione di conflitto di interessi: Gli Autori dichiarano di non avere conflitto di interessi.

Contributi economici degli autori: Gli Autori dichiarano di non aver ricevuto sponsorizzazioni economiche per la preparazione dell'articolo.

Indirizzo degli Autori:

Dr. Giovanni M. Frascà

Nefrologia, Dialisi e Trapianto di rene

Ospedali Riuniti

Via Conca 71

60020 Ancona

giovanni.frasca@ospedaliriuniti.marche.it 


\section{Bibliografia}

1. El-Zoghby ZM, Stegall MD, Lager DJ, et al. Identifying specific causes of kidney allograft loss. Am J Transplant 2009; 9: 527-35.

2. Solez K, Colvin RB, Racusen LC, et al. Banff 07 classification of renal allograft pathology: updates and future directions. Am J Transplant 2008; 8: 753-60.

3. Einecke G, Sis B, Reeve J, et al. Antibody-mediated microcirculation injury is the major cause of late kidney transplant failure. Am J Transplant 2009; 9: 2520-31.

4. Everly MJ, Everly JJ, Arend LJ, et al. Reducing de novo donorspecific antibody levels during acute rejection diminishes renal allograft loss. Am J Transplant 2009; 9: 1063-71.

5. Vangelista A, Frascà GM, Nanni Costa A, Stefoni S, Bonomini $\mathrm{V}$. Value of plasma exchange in renal transplant rejection induced by specific anti-HLA antibodies. Trans Am Soc Artif Intern
Organs 1982; 28: 599-603.

6. Bonomini V, Vangelista A, Frascà GM, Di Felice A, Liviano D'Arcangelo G. Effects of plasmapheresis in renal transplant rejection. A controlled study. Trans Am Soc Artif Intern Organs 1985; 31: 698-703.

7. Frascà GM, Martella D, Vangelista A, Bonomini V. Ten years experience with plasma exchange in renal transplantation. Int $\mathrm{J}$ Artif Organs 1991; 14: 51-5.

8. Ahmed T, Senzel L. The role of therapeutic apheresis in the treatment of acute antibody-mediated kidney rejection. J Clin Apher 2012; 27: 173-7.

9. Singh N, Pirsch J, Samaniego M. Antibody-mediated rejection: treatment alternatives and outcomes. Transplant Rev 2009; 23: 34-46.

10. Waiser J, Budde K, Schutz M, et al. Comparison between bortezomib and rituximab in the treatment of antibody-mediated renal allograft rejection. Nephrol Dial Transplant 2012; 27: 1246-51. 